\title{
Would apixaban be a good option for extended anticoagulation in venous thromboembolism?
}

\author{
Maddalena Alessandra Wu • Giulia Cernuschi
}

Received: 13 May 2013/Accepted: 5 July 2013/Published online: 16 July 2013

(C) SIMI 2013

\section{Background}

The treatment and the long-term management of deep vein thrombosis and pulmonary embolism are a delicate challenging topic. A thoughtful evaluation is warranted to balance the possible risks and benefits of antithrombotic therapy. While there is evidence that anticoagulation can be discontinued after 3 months in most of the patients who experience thromboembolism provoked by precise risk factors [1], conclusive data are still lacking concerning the best treatment regimen for patients with unprovoked venous thromboembolism (VTE), which has a higher rate of recurrence [2].

Some trials address the issue of the possible use in these patients of new anticoagulants, which might be valid alternatives to warfarin due to similar efficacy and administration in fixed doses, without the need for laboratory monitoring.

Apixaban is an oral factor $\mathrm{Xa}$ inhibitor, which has already been shown to be useful for the prevention of stroke in patients with atrial fibrillation (at a dose of $5 \mathrm{mg}$ twice daily) [3] and for thromboprophylaxis after major orthopedic surgery (at a lower dose of $2.5 \mathrm{mg}$ twice daily) [4].

M. A. Wu and G. Cernuschi on behalf of Gruppo di Autoformazione Metodologica (GrAM)

M. A. Wu ( $\varangle) \cdot$ G. Cernuschi

Department of Biomedical and Clinical Sciences, Internal

Medicine III, L. Sacco Hospital, University of Milan, via GB

Grassi, 74, 20157 Milan, Italy

e-mail: folletta@tin.it

G. Cernuschi

e-mail: giulia.cernuschi@gmail.com

\section{Summary}

The randomized, double-blind study by Agnelli et al. [5] compares two doses of apixaban $(2.5$ and $5 \mathrm{mg}$, twice daily) with placebo in patients with symptomatic and objectively confirmed VTE who had completed 6-12 months of anticoagulation therapy, and for whom there was clinical equipoise regarding the continuation or cessation of anticoagulation therapy. Patients were considered eligible if they were 18 years of age or older, and if they had not had a symptomatic recurrence during prior anticoagulant therapy. The main exclusion criteria were ongoing anticoagulant therapy, dual antiplatelet therapy or aspirin at a dose higher than $165 \mathrm{mg}$ daily, a hemoglobin level of less than $9 \mathrm{~g}$ per deciliter, or a serum creatinine level of more than $2.5 \mathrm{mg}$ per deciliter.

Of 2,486 patients randomized, 2,482 were included in the intention-to-treat analyses.

The study was designed to test the hypothesis that each dose of apixaban would be superior to placebo with respect to primary efficacy outcome, assuming an incidence in the placebo group of $6.8 \%$, a power of the study of $90 \%$ and a decrease in the primary outcome of $41 \%$.

The primary efficacy outcome event was the composite of symptomatic recurrent VTE or death from any cause during the 12-month follow-up. It occurred in 96 of the 829 patients who were receiving placebo $(11.6 \%)$, as compared with 32 of the 840 patients $(3.8 \%)$ who were receiving $2.5 \mathrm{mg}$ of apixaban (a difference of 7.8 percentage points-95\% confidence interval 5.5-10.3) and 34 of the 813 patients $(4.2 \%)$ receiving $5 \mathrm{mg}$ of apixaban (a difference of 7.4 percentage points-95\% confidence interval 4.8-10.0); $p<0.001$ for both comparisons. The primary safety outcome was major bleeding, defined as overt bleeding that was associated with a decrease in the 
hemoglobin level of $2 \mathrm{~g}$ per deciliter or more, led to transfusion of 2 or more units of red cells, occurred in a critical site, or contributed to death. The rates of major bleeding were $0.5 \%$ in the placebo group, $0.2 \%$ in the 2.5-mg apixaban group and $0.1 \%$ in the 5-mg apixaban group. The authors conclude that extended anticoagulation with apixaban at either treatment dose $(5 \mathrm{mg})$ or thromboprophylactic dose $(2.5 \mathrm{mg})$ reduces the risk of recurrent VTE without increasing the rate of major bleeding.

\section{Strengths of the study}

- The study was well designed and a large population of patients was enrolled.

- The topic is relevant because it deals with an unresolved issue that is the management of unprovoked VTE.

\section{Weakness of the study}

- Despite the intent to evaluate the superiority of apixaban considering both efficacy and safety, the net clinical benefit includes only major bleeding, such as overt bleeding associated with a decrease in the hemoglobin level of $2 \mathrm{~g}$ per deciliter or more or that occurred in a critical site. Clinically relevant nonmajor bleeding has not been considered.

\section{Question marks}

- In patients with unprovoked VTE-which is considered to be at higher risk - the advantage of apixaban over placebo seems to be predictable. Further relevant points for clinical practice to be addressed in future studies are comparisons of these apixaban doses with other anticoagulants (included warfarin) or acetylsalicylic acid.

- The subgroup analyses were done considering demographic and clinical characteristics of the patients. We wonder if an analysis based on risk profile would have been interesting.

- Some patients prematurely discontinued treatment because of adverse events that are not specified. It might be interesting to know the nature of the events more in detail and if they were directly related to anticoagulation.

- As authors themselves admitted, only $15 \%$ of patients were older than 75 years and few had a body weight below $60 \mathrm{~kg}$ or moderate-severe renal impairment. Further studies should assess the benefit of apixaban in more patients with the afore-mentioned clinical characteristics or with other comorbidities.

- Patients who were lost to follow-up were classified as having had a primary outcome event and as not having had an outcome event for the safety analyses. We wonder if this choice could have affected the results, underestimating the absolute number of adverse events such as bleeding. Nonetheless-considering that in the placebo group there were more patients lost to followup - the estimate of safety would have been in favor of apixaban anyway.

- The authors state that a secondary composite outcome was added after the trial had already begun. Despite the relevance of the results concerning arterial thrombotic events, we wonder if adding a new endpoint during an ongoing trial is appropriate from a methodological point of view.

\section{Sponsorship}

The trial was sponsored by Bristol-Myers Squibb and Pfizer that collected and analyzed data even though the academic authors had access to the data at all times.

\section{Clinical bottom line}

Extended anticoagulation with apixaban can be an option for the prevention of recurrent VTE in specific categories of patients and the thrombotic/hemorrhagic risk profile should be carefully assessed. Further research is needed to directly compare apixaban with warfarin, aspirin and other new anticoagulants.

Conflict of interest None.

\section{References}

1. Torbicki A, Perrier A, Konstantinides S et al (2008) Guidelines on the diagnosis and management of acute pulmonary embolism: the Task Force for the Diagnosis and Management of Acute Pulmonary Embolism of the European Society of Cardiology (ESC). Eur Heart J 29:2276-2315

2. The EINSTEIN Investigators (2010) Oral rivaroxaban for symptomatic venous thromboembolism. N Eng J Med 363:2499-2510

3. Granger CB, Alexander JH, McMurray JJ et al (2011) Apixaban versus warfarin in patients with atrial fibrillation. N Engl J Med 365:981-992

4. Raskob GE, Gallus AS, Pineo GF et al (2012) Apixaban versus enoxaparin for thromboprophylaxis after hip or knee replacement: pooled analysis of major venous thromboembolism and bleeding in 8464 patients from the ADVANCE-2 and ADVANCE-3 trials. J Bone Joint Surg Br 94:257-264

5. Agnelli G, Buller HR, Cohen A et al (2013) Apixaban for extended treatment of venous thromboembolism. N Engl J Med 368:699-708 